\title{
Transport theory within a generalized Boltzmann equation for multiband wave packets
}

\author{
T. Stedman and L. M. Woods $\odot$ \\ Department of Physics, University of South Florida, Tampa, Florida 33620, USA
}

(Received 6 February 2020; revised 19 June 2020; accepted 24 June 2020; published 16 July 2020)

\begin{abstract}
A generalized theory for transport under applied electrochemical potential and temperature gradients that includes multiband effects is presented. This theory relies on a newly defined density operator for multiband wave packets, whose dynamics containing diffusive and scattering contributions are captured in a generalized equation of motion. Given that the multiband energy structure determines the transport explicitly, the case of $N$ degenerate bands within an isotropic constant relaxation time is considered as a particular case for illustration. Electric and thermal currents as well as transport properties are obtained through linear response and comparison to transport in a single band wave packet is provided. Our theory opens avenues for studying novel multiband effects and scattering processes in transport beyond the semiclassical single band approach.
\end{abstract}

DOI: 10.1103/PhysRevResearch.2.033086

\section{INTRODUCTION}

Transport in materials is described assuming that electrons can be treated as free particles whose dispersion is the actual band structure of the material. One typically relies on the semiclassical Boltzmann equation within the standard longwave approximation, for which Bloch electrons are presented as localized wave packets comprised of a single band near the Fermi level $[1,2]$. The theory further relies on the equations of motion for the wave packet center in order to derive various transport properties. Realistic band structures of materials, however, can have several bands near the Fermi level and can contain crossing points or local degeneracies. For example, systems with nontrivial topology, such as Dirac and Weyl materials, have pairs of bands crossing at different points in the Brillouin zone leading to new Hall effect phenomena in transport and magnetism [3-6].

Specifically, the nontrivial topology in materials can lead to an anomalous correction to the group velocity of the Bloch electrons. The anomalous velocity can give rise to a variety of Hall effects associated with charge carriers and photons as well as unusual thermoelectricity [7-11]. On the other hand, several researchers have considered Bloch electron wave packets extending over multiple energy bands [12-16]. In fact, it has been shown that the equations of motion for the centers of such multiband wave packets are different than those in the single band wave packet case, and one can obtain new features in the dynamics of spin, parity polarization currents, and non-Abelian transport [12-16]. The discoveries of Dirac, Weyl, and other materials with nontrivial topology have elevated the importance of interband transitions and coherence of Bloch electrons, which undoubtedly are important

Published by the American Physical Society under the terms of the Creative Commons Attribution 4.0 International license. Further distribution of this work must maintain attribution to the author(s) and the published article's title, journal citation, and DOI. for transport [17-19]. Therefore the semiclassical Boltzmann needs to be re-examined in light of these discoveries.

To this end, research findings strongly suggest that the single band semiclassical transport theory may need to be expanded to capture multiband effects from the materials electronic structure [8,12-17]. This problem has been addressed in recent reports $[8,12,14]$, where a quantum kinetic equation through a Wigner transformation of the Liouville equation for the usual density operator of energy eigenstates is derived. In this way, multiband effects in magnetotransport and various Hall effects can be captured. This approach uses the quasiprobabilistic Wigner distribution function, which in some cases may be negative [20]. Since the standard Boltzmann theory relies on wave packets and the quantum kinetic equation does not, a direct comparison with the Wigner distribution approach may be problematic. Also, to capture multiband effects within the Wigner transformation method one only considers a certain set of bands for the density operator, while the rest of the band structure is ignored without justification. We further note that the quantum kinetic equation has not been applied outside of systems of uniform temperature. It appears that despite the progress made through the quantum kinetic equation, more work is needed to investigate multiband transport under temperature gradients.

Here we develop a new linear transport theory based on a generalized Boltzmann equation for thermoelectric transport in the presence of external fields that explicitly includes multiband effects from the electronic structure of the materials. The theory relies on Bloch electron wave packets, a typical assumption in transport theory concerning processes determined by near equilibrium conditions [1,2]. For our approach, we define a density operator associated with a distribution of wave packets based on the quantum mechanical degrees of freedom in the wave packets. This is a new quantity that uses generalized equations of motion for wave packets containing multiband effects through a previously derived projection procedure [15]. Transport coefficients and related thermoelectric properties are derived within leading orders of the electrochemical potential and temperature gradients to linear 
order of electromagnetic fields in the system. To illustrate the application of this theory, the case of $N$ degenerate bands making up a multiband wave packet is considered. Following basic quantum mechanics, we obtain a generalized tensorlike form of the Boltzmann transport equation for the density operator, transport currents, and thermoelectric properties taking into account the multiband nature of the wave packets, which are directly compared with the single band Boltzmann equation.

\section{BASIC ASSUMPTIONS}

In the Boltzmann equation approach, transport in solids is described by semiclassical wave packets comprised of Bloch electrons. Instead of the usual assumption that transport is due to a single band packet, here we consider multiband packets $\left|\Psi_{\bar{k}}\right\rangle$ over $N$ bands evolving with external electromagnetic fields and temperature gradients present in the periodic environment of the lattice structure of the solids. These wave packets are expressed in the basis of Bloch states $\left|\psi_{n, \boldsymbol{k}}\right\rangle=e^{i \boldsymbol{k} \cdot \boldsymbol{x}}\left|u_{n, \boldsymbol{k}}\right\rangle \quad\left(\left|u_{n, \boldsymbol{k}}\right\rangle\right.$ are the Bloch functions) as $\left|\Psi_{\bar{k}}\right\rangle=\sum_{n=1}^{N} \sum_{\boldsymbol{k} \in I_{N_{\boldsymbol{k}}}} d_{n, \boldsymbol{k}}^{\bar{k}(t)}(t)\left|\psi_{n, \boldsymbol{k}}\right\rangle$ with expansion coefficients $d_{n, \boldsymbol{k}}^{\overline{\boldsymbol{k}}(t)}(t)=c_{n, \boldsymbol{k}}(\overline{\boldsymbol{k}}(t), t) e^{-i \gamma_{n, \boldsymbol{k}}(\overline{\boldsymbol{k}}(t), t)}$ whose magnitudes and phases are denoted as $c_{n, \boldsymbol{k}}(\overline{\boldsymbol{k}}(t), t)$ and $\gamma_{n, \boldsymbol{k}}(\overline{\boldsymbol{k}}(t), t)$, respectively. Note that the magnitudes and phases depend on the band index $n$ ranging over the $N$ bands in the packet and on the wave vector $\boldsymbol{k}$ ranging over a set of $N_{\boldsymbol{k}}$ reciprocal space points centered at $\bar{k}$ with index set $I_{N_{k}}$.

The dynamics of the wave packet in reciprocal space is determined by its center $\overline{\boldsymbol{k}}(t)=\langle\boldsymbol{k}\rangle$ taken as a quantum mechanical average. The total number of Bloch states in the wave packet is then $N_{T}=N * N_{k}$ and the magnitudes have the required normalization $\sum_{n=1}^{N} \sum_{\boldsymbol{k} \in I_{N_{\boldsymbol{k}}}} c_{n, \boldsymbol{k}}^{2}(\overline{\boldsymbol{k}}(t), t)=$ 1. Importantly, the wave packets are assumed to be welllocalized in both reciprocal and real spaces. Specifically, in reciprocal space, we take that $\sum_{\boldsymbol{k} \in I_{N_{k}}} c_{n, \boldsymbol{k}}^{2}(\overline{\boldsymbol{k}}(t), t) h(\boldsymbol{k}) \approx$ $N_{\boldsymbol{k}} c_{n, \overline{\boldsymbol{k}}(t)}^{2}(\overline{\boldsymbol{k}}(t), t) h(\overline{\boldsymbol{k}}(t))$ for any slowly varying function $h(\boldsymbol{k})$, meaning that the spread of the packet is ignored.

The dynamics of the wave packet in real space is determined by its center $\overline{\boldsymbol{x}}(t)$, given as

$$
\begin{aligned}
\overline{\boldsymbol{x}}(t)= & \sum_{n} c_{n}^{2} \nabla_{\overline{\boldsymbol{k}}} \gamma_{n}(\overline{\boldsymbol{k}}(t), t) \\
& +\sum_{m, n} c_{m} c_{n} e^{i\left(\gamma_{m}(\overline{\boldsymbol{k}}(t), t)-\gamma_{n}(\overline{\boldsymbol{k}}(t), t)\right)} \mathcal{A}_{m n}(\overline{\boldsymbol{k}}(t)),
\end{aligned}
$$

which is obtained by using $\left\langle\psi_{m, \boldsymbol{k}}|\boldsymbol{x}| \psi_{n, \boldsymbol{k}^{\prime}}\right\rangle=\delta\left(\boldsymbol{k}-\boldsymbol{k}^{\prime}\right)$ $\left(i \delta_{m n} \nabla_{\boldsymbol{k}}+\mathcal{A}_{m n}(\boldsymbol{k})\right)$, where $\mathcal{A}_{m n}(\boldsymbol{k})=\boldsymbol{i}\left\langle u_{m, \boldsymbol{k}} \mid \nabla_{\boldsymbol{k}} u_{n, \boldsymbol{k}}\right\rangle$ is the interband Berry connection. Both Eq. (1) and the expression for $\left\langle\psi_{m, \boldsymbol{k}}|\boldsymbol{x}| \psi_{n, \boldsymbol{k}^{\prime}}\right\rangle$ treat the wave vector as a continuous quantity. This approximation is valid in the limit of an infinite perfect crystal such that $\frac{1}{V} \sum_{k} \rightarrow \int \frac{d^{3} k}{(2 \pi)^{3}}$.

The localization of wave packets in reciprocal space is a usual assumption in transport theory, since its collapse is determined by the much longer Ehrenfest time compared to the dynamics of the packet even when multiband effects are accounted for [16,21]. The justification for a well-localized packet in real space comes from the semiclassical approximation used in transport for which the wavelengths of external fields are much larger than the spread of the packet in real space. We further note that multiband packets that are well localized in reciprocal and real spaces, one deals with degenerate or nearly degenerate Bloch states comprising the packets $[13,15]$.

We further note that transport theory in materials relies on the density operator $\hat{\rho}$ used to statistically account for possible system states since the exact system wave function is not known. A widely used approach is through Wigner transformation of the Liouville equation $\frac{d \hat{\rho}}{d t}-\frac{i}{\hbar}[\hat{\rho}, H]=0$. In this way, one obtains a quantum kinetic equation for the Wigner function, the Wigner transformed density operator, whose dynamics is determined by the Hamiltonian $H$ of the system $[8,12,14]$. This method uses the standard density operator defined as $\hat{\rho}=\sum_{n} p_{n}\left|\varphi_{n}\right\rangle\left\langle\varphi_{n}\right|$, where $p_{n}$ is the statistical time-independent probability that the system will be in the state $\left|\varphi_{n}\right\rangle$ and $n$ enumerates all of these possible states of the system.

In thermodynamic equilibrium, $\left|\varphi_{n}\right\rangle$ are the Hamiltonian eigenstates with $H\left|\varphi_{n}\right\rangle=E_{n}\left|\varphi_{n}\right\rangle$ and $p_{n}=f\left(E_{n}\right)$ where $f\left(E_{n}\right)=\frac{1}{1+e^{\left(E_{n}-\mu_{T}\right) / k_{B} T}}$ is the Fermi-Dirac probability function with $\mu_{T}=\mu+e V$ being the electrochemical potential $(\mu-$ chemical potential, $V$ - electric potential, $k_{B}$ - the Boltzmann constant, $T$ - temperature). In the one band approximation, the quantum kinetic equation reduces to the standard Boltzmann equation with the Wigner function serving as the Boltzmann distribution function. Given the quasiprobabilistic nature of the Wigner function, however, the correspondence between the quantum kinetic equation and the Boltzmann equation may be unclear.

Density operator for multiband wave packets. In the theory we are developing, a new approach is taken, such that transport is viewed as a result of the dynamics of the Bloch electron multiband wave packets. This is markedly different than the quantum kinetic equation approach, where the concept of a wave packet is not utilized. In our theory, the density operator is based on $\left|\Psi_{\bar{k}}\right\rangle$ and not simply on the energy eigenstates as used in the standard equilibrium density operator above $[8,12,14]$.

This is a new concept, previously unexplored in relation to transport in materials. Such a density operator must account for all possible multiband wave packets through their degrees of freedom captured by the coefficients at $t=0$ with magnitudes $c_{n, \boldsymbol{k}}\left(\overline{\boldsymbol{k}}_{0}, 0\right)=c_{n, \boldsymbol{k}}^{\overline{\boldsymbol{k}}_{0}}$ and phases $\gamma_{n, \boldsymbol{k}}\left(\overline{\boldsymbol{k}}_{0}, 0\right)=\gamma_{n, \boldsymbol{k}}^{\overline{\boldsymbol{k}}_{0}}$ for $\bar{k}(t=0)=\overline{\boldsymbol{k}}_{0}$. These are the initial degrees of freedom before the fields are turned on, since their subsequent values are determined by the Hamiltonian thereafter. The density operator associated with all possible wave packets is then constructed as

$$
\begin{aligned}
\hat{\rho}= & \frac{1}{\operatorname{Tr}\left(\hat{\rho}_{0}\right)} \sum_{\overline{\boldsymbol{k}}_{0}} \iiint \ldots \int \prod_{\boldsymbol{k} \in I_{N_{\boldsymbol{k}}}} d c_{1, \boldsymbol{k}}^{\overline{\boldsymbol{k}}_{0}} \ldots d c_{N, \boldsymbol{k}}^{\overline{\boldsymbol{k}}_{0}} d \gamma_{1, \boldsymbol{k}}^{\overline{\boldsymbol{k}}_{0}} \ldots d \gamma_{N, \boldsymbol{k}}^{\overline{\boldsymbol{k}}_{0}} \\
& \times p\left(\overline{\boldsymbol{k}}\left(c_{1, \boldsymbol{k}}^{\bar{k}_{0}}, \ldots, c_{N, \boldsymbol{k}}^{\bar{k}_{0}}, \gamma_{1, \boldsymbol{k}}^{\overline{\boldsymbol{k}}_{0}} \ldots \gamma_{N, \boldsymbol{k}}^{\overline{\boldsymbol{k}}_{0}}\right),\right. \\
& \left.\times \overline{\boldsymbol{x}}\left(c_{1, \boldsymbol{k}}^{\bar{k}_{0}}, \ldots, c_{N, \boldsymbol{k}}^{\bar{k}_{0}}, \gamma_{1, \boldsymbol{k}}^{\overline{\boldsymbol{k}}_{0}} \ldots \gamma_{N, \boldsymbol{k}}^{\overline{\boldsymbol{k}}_{0}}\right), t\right)\left|\Psi_{\overline{\boldsymbol{k}}}\right\rangle\left\langle\Psi_{\overline{\boldsymbol{k}}}\right| .
\end{aligned}
$$

The above definition includes summation over all possible locations of the wave packets enumerated by their initial locations $\overline{\boldsymbol{k}}_{0}$, for which the coefficients and phases are 
functions of the wave vector $\boldsymbol{k}$ of the Bloch states comprising the wave packets. $\operatorname{Tr}\left(\hat{\rho}_{0}\right)$ is a normalization factor equal to the trace of the equilibrium density operator $\hat{\rho}_{0}$ defined below. By denoting $\eta=\left(c_{1, \boldsymbol{k}}^{\bar{k}_{0}}, \ldots, c_{N, \boldsymbol{k}}^{\bar{k}_{0}}, \gamma_{1, \boldsymbol{k}}^{\bar{k}_{0}} \ldots \gamma_{N, \boldsymbol{k}}^{\overline{\boldsymbol{k}}_{0}}\right)$ and $\mathfrak{D} \eta=$ $\prod_{\boldsymbol{k} \in I_{N_{\boldsymbol{k}}}} d c_{1, \boldsymbol{k}}^{\overline{\boldsymbol{k}}_{0}} \ldots d c_{N, \boldsymbol{k}}^{\overline{\boldsymbol{k}}_{0}} d \gamma_{1, \boldsymbol{k}}^{\overline{\boldsymbol{k}}_{0}} \ldots d \gamma_{N, \boldsymbol{k}}^{\overline{\boldsymbol{k}}_{0}}$, the density operator can be conveniently expressed as

$$
\begin{aligned}
\hat{\rho}= & \frac{1}{\operatorname{Tr}\left(\hat{\rho}_{0}\right)} \sum_{\overline{\boldsymbol{k}}} \int \mathfrak{D} \eta p(\overline{\boldsymbol{k}}(\eta), \overline{\boldsymbol{x}}(\eta), t)\left|\Psi_{\overline{\boldsymbol{k}}}\right\rangle\left\langle\Psi_{\overline{\boldsymbol{k}}}\right| \\
= & \frac{1}{\operatorname{Tr}\left(\hat{\rho}_{0}\right)} \sum_{\overline{\boldsymbol{k}}} \int \mathfrak{D} \eta p(\overline{\boldsymbol{k}}(\eta), \overline{\boldsymbol{x}}(\eta), t) c_{m, \boldsymbol{k}}(\overline{\boldsymbol{k}}(t), t) \\
& \times c_{n, \boldsymbol{k}^{\prime}}^{*}(\overline{\boldsymbol{k}}(t), t)\left|\psi_{m, \boldsymbol{k}}\right\rangle\left\langle\psi_{n, \boldsymbol{k}^{\prime}}\right|,
\end{aligned}
$$

where summation over repeating indices $m, n$ and wave vectors $\boldsymbol{k}, \boldsymbol{k}^{\prime}$ is implied. Also, $\int \mathfrak{D} \eta$ is understood as a multiple integral whose dimension is equal to twice the total number of Bloch states (accounting for magnitudes and phases) comprising the wave packet, as given in Eq. (2). This multidimensional integration is subject to the constraint $\sum_{n=1}^{N} \sum_{k \in I_{N_{\boldsymbol{k}}}}\left(c_{n, \boldsymbol{k}}^{\overline{\boldsymbol{k}}_{0}}\right)^{2}=1$, which confines these magnitudes to the surface area of a unit sphere in the positive quadrant of a space with dimension $N_{T}$. The integration interval of the phases $\gamma_{n, \boldsymbol{k}}^{\bar{k}_{0}}$ is $[0,2 \pi]$.

The density operator in Eqs. (2) and (3) relies on the time-dependent probability $p(\overline{\boldsymbol{k}}(\eta), \overline{\boldsymbol{x}}(\eta), t)$ which denotes the system probability distribution for the wave packets $\left|\Psi_{\bar{k}}\right\rangle$. The probability depends on $\overline{\boldsymbol{k}}$ and $\overline{\boldsymbol{x}}$ of all possible packets since these are well-localized in both reciprocal and real spaces. In equilibrium, one has $p(\overline{\boldsymbol{k}}(\eta), \overline{\boldsymbol{x}}(\eta), t)=f(\overline{\mathcal{E}}(\overline{\boldsymbol{k}}))$ with $\overline{\mathcal{E}}(\overline{\boldsymbol{k}})$ being the equilibrium energy of each packet. The explicit time dependence is also included in $p(\overline{\boldsymbol{k}}(\eta), \overline{\boldsymbol{x}}(\eta), t)$ to account for scattering events [1,2]. Unlike the Wigner function, $p(\overline{\boldsymbol{k}}(\eta), \overline{\boldsymbol{x}}(\eta), t)$ is associated with the multiband wave packet and has a truly probabilistic nature.

We further note that the equilibrium density operator $\hat{\rho}_{0}$ for the wave packets is $\hat{\rho}_{0}=\sum_{\bar{k}} \int \mathfrak{D} \eta f(\overline{\mathcal{E}})\left|\Psi_{\bar{k}}\right\rangle\left\langle\Psi_{\bar{k}}\right|$. Although the wave packets are not eigenstates of the unperturbed crystal Hamiltonian $H^{0}$, this expression for $\hat{\rho}_{0}$ is equivalent up to a factor $\operatorname{Tr}\left(\hat{\rho}_{0}\right)$ to the operator one obtains if the Bloch energy eigenstates are used [22]. This treatment of $\hat{\rho}_{0}$ is consistent with the formulation of the more general $\hat{\rho}$ in Eq. (3). Upon integrating over the degrees of freedom, we find $\operatorname{Tr}\left(\hat{\rho}_{0}\right)=$ $\frac{1}{N} \pi^{N_{T}} S_{N_{T}}$, where $S_{N_{T}}=\frac{2 \pi \frac{N_{T}}{2}}{\Gamma\left(\frac{N_{T}}{2}\right)}$ is the surface area of a unit sphere in $N_{T}$-dimensional space. Importantly, $\operatorname{Tr}(\hat{\rho})=1$ for any density operator $\hat{\rho}$ and so $\operatorname{Tr}\left(\hat{\rho}_{0}\right)$ represents a normalization factor needed in the density operator in Eqs. (2), (3) for this linear response theory to linear order of the electromagnetic fields and temperature gradients.

Steady state for the packet density operator. Using Eq. (3), the steady-state equation for the wave packet density operator is obtained as

$$
\frac{d \hat{\rho}}{d t}=\hat{\rho}_{\dot{\overline{\boldsymbol{k}}}}+\hat{\rho}_{\dot{\overline{\boldsymbol{x}}}}-\frac{\partial \hat{\rho}_{S}}{\partial t}+\frac{i}{\hbar}[\hat{\rho}, H]=0 .
$$

The first two terms in the above expression come from the drift motion of the wave packet centers in reciprocal and real spaces. The last two terms are reminiscent of a Liouville-like equation, where $\frac{\partial \hat{\rho}_{S}}{\partial t}$ accounts for the scattering processes while the commutator involves the full density operator and full Hamiltonian of the system.

One notes that $\hat{\rho}_{\dot{\bar{k}}}, \hat{\rho}_{\dot{\bar{x}}}$, and $\frac{\partial \hat{\rho}_{S}}{\partial t}$ are associated with the dynamics of the probability distribution function $p(\overline{\boldsymbol{k}}, \overline{\boldsymbol{x}}, t)$ for the wave packet localized at $\overline{\boldsymbol{k}}$ and $\overline{\boldsymbol{x}}$, whose local equilibrium rate of change in time is given by the typical relation $\frac{d p}{d t}=$ $\nabla_{\overline{\boldsymbol{k}}} f(\overline{\boldsymbol{k}}, \overline{\boldsymbol{x}}) \cdot \dot{\overline{\boldsymbol{k}}}+\nabla_{\overline{\boldsymbol{x}}} f(\overline{\boldsymbol{k}}, \overline{\boldsymbol{x}}) \cdot \dot{\overline{\boldsymbol{x}}}+\frac{\partial p_{S}}{\partial t}$. The drift terms coming from $\dot{\overline{\boldsymbol{k}}}$ and $\dot{\overline{\boldsymbol{x}}}$ are determined by the Fermi distribution function $f(\overline{\boldsymbol{k}}, \overline{\boldsymbol{x}})=\frac{1}{1+e^{\left(\mathcal{E}(\bar{k})-\mu_{T}(\overline{\boldsymbol{x}}) / k_{B} T(\overline{\boldsymbol{x}})\right.}}$, where $\overline{\mathcal{E}}(\overline{\boldsymbol{k}})$ is the energy of the wave packet center in reciprocal space, $\mu_{T}(\overline{\boldsymbol{x}})$ is the electrochemical potential and $T(\bar{x})$ is the temperature. We emphasize that the description of the dynamics in Eq. (4) and the Fermi distribution function are for well-localized packets whose spreads in reciprocal and real spaces are negligible as discussed earlier. Explicitly,

$$
\begin{aligned}
\hat{\rho}_{\dot{\overline{\boldsymbol{k}}}} & =\frac{1}{\operatorname{Tr}\left(\hat{\rho}_{0}\right)} \sum_{\overline{\boldsymbol{k}}} \int \mathfrak{D} \eta \nabla_{\overline{\boldsymbol{k}}} f(\overline{\boldsymbol{k}}(\eta), \overline{\boldsymbol{x}}(\eta)) \cdot \dot{\overline{\boldsymbol{k}}}\left|\Psi_{\overline{\boldsymbol{k}}}\right\rangle\left\langle\Psi_{\overline{\boldsymbol{k}}}\right|, \\
\hat{\rho}_{\dot{\overline{\boldsymbol{x}}}} & =\frac{1}{\operatorname{Tr}\left(\hat{\rho}_{0}\right)} \sum_{\overline{\boldsymbol{k}}} \int \mathfrak{D} \eta \nabla_{\overline{\boldsymbol{x}}} f(\overline{\boldsymbol{k}}(\eta), \overline{\boldsymbol{x}}(\eta)) \cdot \dot{\overline{\boldsymbol{x}}}\left|\Psi_{\overline{\boldsymbol{k}}}\right\rangle\left\langle\Psi_{\overline{\boldsymbol{k}}}\right|, \\
\frac{\partial \hat{\rho}_{S}}{\partial t} & =-\frac{1}{\operatorname{Tr}\left(\hat{\rho}_{0}\right)} \sum_{\overline{\boldsymbol{k}}} \int \mathfrak{D} \eta \frac{\partial p(\overline{\boldsymbol{k}}(\eta), \overline{\boldsymbol{x}}(\eta), t)}{\partial t}\left|\Psi_{\overline{\boldsymbol{k}}}\right\rangle\left\langle\Psi_{\overline{\boldsymbol{k}}}\right| .
\end{aligned}
$$

The scattering term $\frac{\partial \hat{\rho}_{S}}{\partial t}$ depends on the particular scattering mechanisms and is complicated in general [1,2]. To better illustrate the electronic structure effects on the multiband wave packet in this new theory, we work within the relaxation time approximation $\frac{\partial \hat{\rho}_{S}}{\partial t}=-\frac{1}{2}\left\{\hat{\rho}-\hat{\rho}_{0}, \hat{\tau}^{-1}\right\}=$ where $\hat{\tau}^{-1}$ is the inverse of the scattering time operator $\hat{\tau}(\boldsymbol{k})$. We can then express the density operator from Eq. (4) as

$$
\hat{\rho}=-\hat{\tau} \hat{\rho} \hat{\tau}^{-1}+\hat{\tau}\left\{\hat{\rho}_{0}, \hat{\tau}^{-1}\right\}-2 \hat{\tau}\left(\frac{i}{\hbar}[\hat{\rho}, H]+\hat{\rho}_{\dot{\overline{\boldsymbol{k}}}}+\hat{\rho}_{\dot{\overline{\boldsymbol{x}}}}\right) .
$$

Note that the commutator $[\hat{\rho}, H]$ can be obtained in the case of static fields for which $H$ is time-independent. This can be done by using the evolution operator $U(t)=e^{-i H t / \hbar}$ realizing that $[\hat{\rho}, H]=e^{-\frac{i}{\hbar} H t}[\hat{\rho}(t=0), H] e^{\frac{i}{\hbar} H t}$. Since we use linear response transport theory, only terms within linear order of electromagnetic fields and gradients are considered. The Hamiltonian in the presence of electromagnetic fields can be expressed as $H \approx H^{0}+H^{1}$, where $H^{0}$ is the bare unperturbed crystal Hamiltonian and $H^{1}$ is the first term of a real space Taylor expansion of the full Hamiltonian about the wave packet centers $\overline{\boldsymbol{x}}[3,5-7,15]$. Using the explicit expressions for the perturbative series of the Hamiltonian, we find that since $\hat{\rho}(t=0)$ is diagonal in the Bloch states to zeroth order, 
then

$$
\begin{aligned}
{[\hat{\rho}(t=0), H]=} & {\left[\hat{\rho}_{0}, H^{1}\right] } \\
= & -\frac{e}{\hbar} \boldsymbol{E}_{T} \cdot \sum_{\boldsymbol{k}} \int \mathfrak{D} \eta\left[\nabla_{\boldsymbol{k}}(f(\mathcal{E}(\boldsymbol{k}))\right. \\
& \left.\left.-\frac{\partial f}{\partial \mathcal{E}(\boldsymbol{k})}\langle\hat{\boldsymbol{M}} \cdot \boldsymbol{B}\rangle\right) c_{m}^{0}(\boldsymbol{k})^{2}\right]\left|\psi_{m, \boldsymbol{k}}\right\rangle\left\langle\psi_{m, \boldsymbol{k}}\right|
\end{aligned}
$$

where $e \boldsymbol{E}_{T}=-\nabla_{\overline{\boldsymbol{x}}} \mu_{T}(\overline{\boldsymbol{x}})$ is constant and $\hat{\boldsymbol{M}}$ is the wave packet magnetization with Bloch state components $\hat{\boldsymbol{M}}_{m n}(\boldsymbol{k})=$ $-\frac{i e}{2 \hbar}\left\langle\nabla_{\boldsymbol{k}} u_{m}\left|\left(\mathcal{E}(\boldsymbol{k})-\hat{H}_{0}\right) \times\right| \nabla_{\boldsymbol{k}} u_{n}\right\rangle$. Details from the calculations can be found in Ref. [22].

Equation (4) is the main transport equation for multiband wave packets with the newly constructed density operator in Eq. (3). Equation (6) is the transport equation for the multiband packet in the relaxation time approximation. The corresponding relation for a single band wave packet is the steady-state semiclassical Boltzmann equation

$$
\nabla_{\overline{\boldsymbol{k}}} f_{B}(\overline{\boldsymbol{k}}, \overline{\boldsymbol{x}}, t) \cdot \dot{\overline{\boldsymbol{k}}}+\nabla_{\overline{\boldsymbol{x}}} f_{B}(\overline{\boldsymbol{k}}, \overline{\boldsymbol{x}}, t) \cdot \dot{\overline{\boldsymbol{x}}}+\frac{\partial f_{B}(\overline{\boldsymbol{k}}, \overline{\boldsymbol{x}}, t)}{\partial t}=0,
$$

where $f_{B}(\overline{\boldsymbol{k}}, \overline{\boldsymbol{x}}, t)$ is the Boltzmann distribution function. Comparing Eqs. (4) and (8) shows that there are similar diffusion and scattering terms for the single and multiband wave packets, however there is an extra commutator term in Eq. (4) due to the use of the newly defined density operator. The density operator in Eq. (4) plays the role of $f_{B}(\overline{\boldsymbol{k}}, \overline{\boldsymbol{x}}, t)$ in Eq. (8). Distinguishing further, we note that the Boltzmann equation uses a semiclassical phase space of the single band wave packet centers $\overline{\boldsymbol{x}}$ and $\overline{\boldsymbol{k}}$, whereas the density operator $\hat{\rho}$ from Eq. (3) yields a phase space based on the initial magnitudes $c_{n, \boldsymbol{k}}^{\bar{k}_{0}}$ and phases $\gamma_{n, \boldsymbol{k}}^{\bar{k}_{0}}$ of expansion coefficients of the multiband wave packets in addition to $\overline{\boldsymbol{k}}$.

The theory we have developed is an alternative approach to the more established Wigner formalism for quantum kinetic equations. In the Wigner approach, a finite set of quantum mechanical states of the system Hamiltonian are accounted for in the density operator [20]. One notes that there is no justification for disregarding the rest of the states of the Hamiltonian. Also, the Wigner transformed density operator function can have negative values, thus its direct connection to the Boltzmann transport equation, which relies on a probabilistic distribution, may be problematic. In our approach, band structure effects are taken into account through the construction of a density operator of multiband wave packets, which in essence are semiclassical entities. Such a theory extends seamlessly to the standard Boltzmann transport equation beyond the single band wave packet, as it includes multiband features in the transport. Our approach can be regarded as a complementary to the Wigner approach, which although well equipped to study various quantum effects, has not been applied to transport phenomena under temperature gradients.

\section{TRANSPORT IN $\boldsymbol{N}$ DEGENERATE BANDS}

Equations (2)-(5c) constitute the main theoretical framework for our transport theory of wave packets. The newly defined density operator is time dependent and its degrees of freedom set by general multiband wave packets allow for a phase space description of a generalized quantum mechanical transport equation. The steady state in Eq. (4) depends on the dynamics of the wave packets through the equations of motion of $\dot{\overline{\boldsymbol{k}}}$ and $\dot{\overline{\boldsymbol{x}}}$. In general, the equations of motion for a multiband wave packet are complicated [15]. These relations can be found through Hamiltonian dynamics with operators projected onto the wave packet subspace while using a perturbed minimally coupled electromagnetic Hamiltonian through a Taylor expansion about the wave packet centers in real space [13,15,23]. As discussed in [15], the projection procedure is justified from adiabatic conditions when the $N$ bands of the packet are separated by large energy gaps from the rest of the band structure.

To facilitate our calculations further, we take the specific case of $N$ degenerate bands of energy dispersion $\mathcal{E}$ making up the wave packet within the isotropic relaxation scattering time $\hat{\tau}(\boldsymbol{k})=\hat{I} \tau(\boldsymbol{k})$. Within this particular case, multiband effects are emphasized without additional complications coming from interband scattering mechanisms. For such a band structure, the equations of motion for $\dot{\overline{\boldsymbol{x}}}$ and $\dot{\overline{\boldsymbol{k}}}$ are found to be [13]

$$
\dot{\overline{\boldsymbol{x}}}=\left\langle\left[\hat{\boldsymbol{D}}, \hat{\mathcal{E}}_{M}\right]-\dot{\overline{\boldsymbol{k}}} \times \hat{\mathcal{F}}\right\rangle, \quad \dot{\overline{\boldsymbol{k}}}=e\left(\boldsymbol{E}_{T}+\langle\hat{\boldsymbol{v}}\rangle \times \boldsymbol{B}\right) .
$$

Here $\langle\hat{\boldsymbol{v}}\rangle=\frac{1}{\hbar}\left\langle\nabla_{\boldsymbol{k}} \mathcal{E}(\boldsymbol{k})\right\rangle=\frac{1}{\hbar} \nabla_{\overline{\boldsymbol{k}}} \mathcal{E}(\overline{\boldsymbol{k}})$ is the standard group velocity $\hat{\boldsymbol{v}}(\boldsymbol{k})=\frac{1}{\hbar} \nabla_{\boldsymbol{k}} \mathcal{E}(\boldsymbol{k})$ of the packet for the $N$ degenerate bands of dispersion $\mathcal{E}(\boldsymbol{k})$ evaluated at the reciprocal space center $\overline{\boldsymbol{k}}$ and $\hat{\mathcal{E}}_{M}(\boldsymbol{k})=\hat{I} \mathcal{E}(\boldsymbol{k})-\hat{\boldsymbol{M}}(\boldsymbol{k}) \cdot \boldsymbol{B}$ is the generalized crystal Hamiltonian operator with a magnetization contribution from the wave packet magnetization $\hat{\boldsymbol{M}}_{m n}(\boldsymbol{k})=$ $-\frac{i e}{2 \hbar}\left\langle\nabla_{\boldsymbol{k}} u_{m}\left|\left(\mathcal{E}(\boldsymbol{k})-\hat{H}_{0}\right) \times\right| \nabla_{\boldsymbol{k}} u_{n}\right\rangle$ coupled to the magnetic field $\boldsymbol{B}$. Also, $\hat{\boldsymbol{D}}(\boldsymbol{k})=\hat{I} \nabla_{\boldsymbol{k}}-i \hat{\mathcal{A}}$ is the covariant derivative operator and $\hat{\mathcal{F}}(\boldsymbol{k})=\hat{\mathbf{\Omega}}(\boldsymbol{k})+i \hat{\boldsymbol{Q}}(\boldsymbol{k})$ is the non-Abelian gauge field whose components are defined from $\hat{\mathcal{F}}_{i}=\frac{1}{2} \epsilon_{i j k} \hat{\mathcal{F}}_{j k}$ with $\hat{\mathcal{F}}_{j k}=\left[\hat{D}_{j}, \hat{D}_{k}\right]$. For $\hat{\mathcal{F}}$, the interband Berry curvature $\hat{\mathbf{\Omega}}=$ $\nabla_{\boldsymbol{k}} \times \hat{\mathcal{A}}$ is used with components defined from $\hat{\Omega}_{i}=\frac{1}{2} \epsilon_{i j k} \hat{\Omega}_{j k}$ and $\hat{\Omega}_{j k}=\partial_{j} \hat{\mathcal{A}}_{k}-\partial_{k} \hat{\mathcal{A}}_{j}$ along with the non-Abelian contribution $\hat{\boldsymbol{Q}}$ whose components are defined from $\hat{Q}_{i}=\frac{1}{2} \epsilon_{i j k} \hat{Q}_{j k}$ with $\hat{Q}_{j k}=\hat{\mathcal{A}}_{j} \hat{\mathcal{A}}_{k}-\hat{\mathcal{A}}_{k} \hat{\mathcal{A}}_{j}$. Additionally, using Eq. (9) we identify $\hat{\boldsymbol{v}}_{G}=\frac{1}{\hbar}\left[\hat{\boldsymbol{D}}, \hat{\mathcal{E}}_{M}\right]-\dot{\overline{\boldsymbol{k}}} \times \hat{\mathcal{F}}$ as the generalized velocity operator for these degenerate band wave packets. The equations of motion for the $N$ degenerate band wave packet in Eq. (9) are gauge invariant under a gauge transformation $\left|u_{n}^{\prime}(\boldsymbol{k})\right\rangle=\sum_{m}\left|u_{m}(\boldsymbol{k})\right\rangle g_{m n}(\boldsymbol{k})$ of the basis Bloch functions with $g^{\dagger} g=1$, as also noted by others in earlier reports $[8,13]$.

Currents and transport properties. The framework of the transport theory for multiband wave packets within the isotropic relaxation time approximation and the chosen specific band structure enables us to calculate transport currents and properties. For this purpose, we utilize the quantum mechanical operator definitions of the operators for the electric current $\hat{\boldsymbol{J}}$ and heat current $\hat{\boldsymbol{J}}_{Q}$ generalized from their classical mechanics expressions $[23,24]$,

$$
\hat{\boldsymbol{J}}=\rho_{e} \hat{\boldsymbol{v}}_{G}, \quad \hat{\boldsymbol{J}}_{Q}=\frac{1}{2 e}\left\{\hat{\mathcal{E}}_{M}, \hat{\boldsymbol{J}}\right\}-\frac{\mu_{T}}{e} \hat{\boldsymbol{J}},
$$

where $\rho_{e}$ is the uniform free charge density of the material, $\hat{\boldsymbol{v}}_{G}=\frac{1}{\hbar}\left[\hat{\boldsymbol{D}}, \hat{\mathcal{E}}_{M}\right]-\dot{\overline{\boldsymbol{k}}} \times \hat{\mathcal{F}}$ is the generalized velocity operator [see Eq. (9)], and $\left\{\hat{\mathcal{E}}_{M}, \hat{\boldsymbol{J}}\right\}=\hat{\mathcal{E}}_{M} \hat{\boldsymbol{J}}+\hat{\boldsymbol{J}} \hat{\mathcal{E}}_{M}$. As known 
TABLE I. The integrals $\overleftrightarrow{\mathcal{L}}^{(\alpha)}$ and $\overleftrightarrow{\mathcal{L}}_{G}^{(\alpha)}$ for single band wave packets and multiband wave packets of $\boldsymbol{N}$ degenerate bands respectively, used to define the linear transport coefficients $\overleftrightarrow{\boldsymbol{L}}_{11}, \overleftrightarrow{\boldsymbol{L}}_{12}, \overleftrightarrow{\boldsymbol{L}}_{21}$, and $\overleftrightarrow{\boldsymbol{L}}_{22}$ for the electric and heat currents. Here $\boldsymbol{T r}_{N}$ denotes a trace over the $\boldsymbol{N}$ degenerate bands of the multiband wave packet and $\otimes$ denotes a tensor product. Also, $\hat{\mathcal{E}}_{\boldsymbol{M}}(\boldsymbol{k})=\hat{\boldsymbol{I}} \mathcal{E}(\boldsymbol{k})-\hat{\boldsymbol{M}}(\boldsymbol{k}) \cdot \boldsymbol{B}$ and $\hat{\boldsymbol{v}}_{\boldsymbol{G}}=\frac{1}{\hbar}\left[\hat{\boldsymbol{D}}, \hat{\mathcal{E}}_{\boldsymbol{M}}\right]-\dot{\overline{\boldsymbol{k}}} \times \hat{\mathcal{F}}$ are magnetization energy and generalized group velocity, respectively, and they are defined in the text.

\begin{tabular}{|c|c|c|c|c|}
\hline \multirow[t]{2}{*}{ Transport integrals } & \multicolumn{4}{|c|}{$\begin{array}{c}\stackrel{\leftrightarrow}{\mathcal{L}}^{(\alpha)}=N_{e} e^{2} \int \frac{d^{3} \boldsymbol{k}}{4 \pi^{3}} \tau(\boldsymbol{k})\left(-\frac{\partial f}{\partial \mathcal{E}}\right)\left(\mathcal{E}-\mu_{T}\right)^{\alpha} \boldsymbol{v} \otimes \boldsymbol{v} \\
\overleftrightarrow{\mathcal{L}}_{G}^{(\alpha)}=N_{e} e^{2} \int \frac{d^{3} \boldsymbol{k}}{4 \pi^{3}} \tau(\boldsymbol{k})\left(-\frac{\partial f}{\partial \mathcal{E}}\right) \operatorname{Tr}_{\mathrm{N}}\left(\left(\hat{\mathcal{E}}_{M}-\mu_{T}\right)^{\alpha} \hat{\boldsymbol{v}}_{G} \otimes \hat{\boldsymbol{v}}_{G}\right)\end{array}$} \\
\hline & $\stackrel{\leftrightarrow}{L}_{11}$ & $\stackrel{\leftrightarrow}{L}_{12}$ & $\stackrel{\leftrightarrow}{L}_{21}$ & $\overleftrightarrow{L}_{22}$ \\
\hline Single band wave packet & $\overleftrightarrow{\mathcal{L}}^{(0)}$ & $\frac{1}{e T} \stackrel{\leftrightarrow}{\mathcal{L}}^{(1)}$ & $\frac{1}{e} \overleftrightarrow{\mathcal{L}}^{(1)}$ & $\frac{1}{e^{2} T} \stackrel{\leftrightarrow}{\mathcal{L}}^{(2)}$ \\
\hline Multiband wave packet with $N$ degenerate bands & $\overleftrightarrow{\mathcal{L}}_{G}^{(0)}$ & $\frac{1}{e T} \stackrel{\leftrightarrow}{\mathcal{L}}_{G}^{(1)}$ & $\frac{1}{e} \stackrel{\leftrightarrow}{\mathcal{L}}_{G}^{(1)}$ & $\frac{1}{e^{2} T} \overleftrightarrow{\mathcal{L}}_{G}^{(2)}$ \\
\hline
\end{tabular}

from quantum mechanics [16,21], the measured currents are determined by the traces $\operatorname{Tr}(\hat{\rho} \hat{\boldsymbol{J}})$ and $\operatorname{Tr}\left(\hat{\rho} \hat{\boldsymbol{J}}_{Q}\right)$. Since $\hat{\boldsymbol{J}}$ is diagonal in the wave vector and $\hat{\tau}=\hat{I} \tau(\boldsymbol{k})$ for degenerate bands, $\operatorname{Tr}\left(\hat{\tau} \hat{\rho} \hat{\tau}^{-1} \hat{\boldsymbol{J}}\right)=\operatorname{Tr}(\hat{\rho} \hat{\boldsymbol{J}})$ and Eq. (6) can be used to solve for $\operatorname{Tr}(\hat{\rho} \hat{\jmath})$. A similar analysis can be applied to the heat current $\hat{\boldsymbol{J}}_{Q}$, which is also diagonal in the wave vector, to solve for $\operatorname{Tr}\left(\hat{\rho} \hat{\boldsymbol{J}}_{Q}\right)$. Using Eqs. (6) and (10), after integrating over all degrees of freedom one finds [22],

$$
\begin{aligned}
\operatorname{Tr}(\hat{\rho} \hat{\boldsymbol{J}})= & e \rho_{e} \operatorname{Tr}\left\{\hat{\tau}\left(-\frac{\partial f}{\partial \mathcal{E}}\right) \hat{\boldsymbol{v}}_{G}\right. \\
& \left.\times\left[\hat{\boldsymbol{v}}_{G} \cdot\left(\boldsymbol{E}_{T}-\frac{1}{e}\left(\frac{\hat{\mathcal{E}}_{M}-\mu_{T}}{T}\right) \nabla_{\overline{\boldsymbol{x}}} T\right)\right]\right\}, \\
\operatorname{Tr}\left(\hat{\rho} \hat{\boldsymbol{J}}_{Q}\right)= & \rho_{e} \operatorname{Tr}\left\{\hat{\tau}\left(-\frac{\partial f}{\partial \mathcal{E}}\right)\left(\hat{\mathcal{E}}_{M}-\mu_{T}\right) \hat{\boldsymbol{v}}_{G}\right. \\
& \left.\times\left[\hat{\boldsymbol{v}}_{G} \cdot\left(\boldsymbol{E}_{T}-\frac{1}{e}\left(\frac{\hat{\mathcal{E}}_{M}-\mu_{T}}{T}\right) \nabla_{\overline{\boldsymbol{x}}} T\right)\right]\right\},
\end{aligned}
$$

By imposing linear response requirements, Eqs. (11a) and (11b) give the terms proportional to the potential and temperature gradients up to linear order of the electromagnetic fields as required by the perturbative Hamiltonian used for the generalized equations of motion [22].

The above relations are the quantum mechanically and statistically averaged linear response currents, which can be put in the familiar form

$$
\begin{aligned}
\operatorname{Tr}(\hat{\rho} \hat{\boldsymbol{J}}) & =\overleftrightarrow{L}_{11} \boldsymbol{E}_{T}-\overleftrightarrow{L}_{12} \nabla T, \\
\operatorname{Tr}\left(\hat{\rho} \hat{\boldsymbol{J}}_{Q}\right) & =\overleftrightarrow{L}_{21} \boldsymbol{E}_{T}-\overleftrightarrow{L}_{22} \nabla T
\end{aligned}
$$

where the coefficients $\overleftrightarrow{L}_{11}, \overleftrightarrow{L}_{12}, \overleftrightarrow{L}_{21}, \overleftrightarrow{L}_{22}$ are Cartesian tensors. From here the electric conductivity $\overleftrightarrow{\sigma}$, Seebeck coefficient $\overleftrightarrow{S}$, and thermal conductivity $\overleftrightarrow{\kappa}$ can be extracted to complete the description of the transport as

$$
\overleftrightarrow{\sigma}=\overleftrightarrow{L}_{11}, \overleftrightarrow{S}=\overleftrightarrow{L}_{11}^{-1} \stackrel{\leftrightarrow}{L}_{12}, \overleftrightarrow{\kappa}=\overleftrightarrow{L}_{22}-\overleftrightarrow{L}_{21} \stackrel{\leftrightarrow}{L}_{11}^{-1} \overleftrightarrow{L}_{12}
$$

The subsequent results are gathered in Table I. For comparison, we also give the corresponding quantities for transport of a wave packet comprised of a single band. The results in Table I for the single band case follow from the semiclassical Boltzmann Eq. (8) in the isotropic relaxation time approximation. Using Table I, the explicit expressions for the transport properties in Eqs. (13) can be obtained to linear order of the applied fields and gradients and these are organized in Table II. For $\overleftrightarrow{\kappa}$, we used the approximation $\overleftrightarrow{\kappa} \approx \overleftrightarrow{L}_{22}$ consistent with the Wiedemann-Franz relation.

At this point it is instructive to compare the transport coefficients for the $N$ degenerate multiband case to the single band semiclassical Boltzmann case (Tables I and II). The overall appearance of the characteristic transport integrals for both cases is similar. A notable distinction, however, is that the group velocity $\overline{\boldsymbol{v}}$ and energy $\mathcal{E}$ of the single band packet are promoted to their multiband operator equivalents $\hat{\boldsymbol{v}}_{G}$ and $\hat{\mathcal{E}}_{M}$, respectively. This observation is reminiscent of the promotion of observables to operators in transitioning from classical to quantum physics. The transport coefficients in Eqs. (13) are found by trace averaging, converting these operators to scalar observables [24,25].

Interestingly, the differences between the results in Tables I and II for the single band semiclassical Boltzmann case as compared to those for the $N$ degenerate multiband case are distinguished in the presence of the magnetic field $\boldsymbol{B}$. When there is no $\boldsymbol{B}$ field, the system does not perceive the $N$ degenerate bands as separate bands but rather as one whole band. This is in line with the isotropic relaxation time approximation in which no interband transitions would take place between states of equal wave vector for a system of degenerate bands. However, new terms due to coupling of the anomalous velocity and magnetization with the magnetic field appear in the properties, as evident in Table I and II, suggesting that a $\boldsymbol{B}$ field is required to probe these new effects in the transport. Nevertheless, because of the $T r$ operator, these interband contributions are essentially washed away and what remains is a sum of the group and anomalous velocity contributions. This result is similar to the transport properties obtained with the standard Boltzmann equation for a single band packet when the anomalous velocity is probed via an external magnetic field. The numerical magnitude of these new multiband effects depends on the anomalous part of the generalized velocity, the Berry connection, and the magnetization, which are material dependent properties. One needs to know the energy band structure (either from ab initio calculations or parametrized analytical models) of a particular material, which then have to be implemented in this theory. While this certainly goes beyond the scope of the present study, we hope the paper inspires research to be focused on the material-dependent aspect of multiband transport. 
TABLE II. The conductivity, Seebeck coefficient, and thermal conductivity for $\boldsymbol{N}$ degenerate band wave packets. The explicit dependence of the operator $\hat{\boldsymbol{v}}_{\boldsymbol{B}}$ is given through the magnetization $\hat{\boldsymbol{M}}$, the non-Abelian gauge field $\hat{\mathcal{F}}$, and the interband Berry connection $\hat{\mathcal{A}}$ (all specified in matrix component form).

\begin{tabular}{lr}
\hline \hline Transport properties & $\hat{\boldsymbol{v}}_{\boldsymbol{B}}=\frac{1}{\hbar}\left(\nabla_{\boldsymbol{k}}(\hat{\boldsymbol{M}} \cdot \boldsymbol{B})+e(\boldsymbol{v} \times \boldsymbol{B}) \times \hat{\mathcal{F}}\right) ; \hat{\boldsymbol{M}}_{m n}(\boldsymbol{k})=-\frac{i e}{2 \hbar}\left\langle\nabla_{\boldsymbol{k}} u_{m, \boldsymbol{k}}\left|\left(\mathcal{E}(\boldsymbol{k})-\hat{H}_{0}\right) \times\right| \nabla_{\boldsymbol{k}} u_{n, \boldsymbol{k}}\right\rangle$ \\
$\hat{\mathcal{F}}_{m n}(\boldsymbol{k})=\left(\nabla_{\boldsymbol{k}} \times \hat{\mathcal{A}}\right)_{m n}+i(\hat{\mathcal{A}} \times \hat{\mathcal{A}})_{m n} ; \hat{\mathcal{A}}_{m n}(\boldsymbol{k})=\left\langle i u_{m, \boldsymbol{k}} \mid \nabla_{\boldsymbol{k}} u_{n, \boldsymbol{k}}\right\rangle$
\end{tabular}

\section{DISCUSSIONS AND CONCLUSIONS}

The transport theory developed here concerns processes under the application of external voltage and temperature gradients when multiple bands from the electronic structure of the materials are important. This approach is a quantum mechanical generalization of the main assumptions and quantities of the traditional semiclassical theory for single band transport. Specifically, a density operator for a multiband wave packet distribution is defined by enforcing its dependence on the degrees of freedom specifying the packets. In the semiclassical single band approach, a distribution function based on a classical phase space of the single band wave packets is used instead of a density operator. A generalized transport equation for the density operator is also given, which now uses generalized velocity and Hamiltonian operators based on the dynamics of the multiband wave packets.

In order to see how electronic structure effects beyond the single band case come into play, we explicitly consider $N$ degenerate bands making up the wave packet. This energy dispersion enters explicitly in the generalized Boltzmann equation and through the equations of motion of the wave packet centers in reciprocal and real spaces, which have been found previously by a perturbative Hamiltonian expansion and projection procedure. Using the general framework of the theory together with the specific band structure choice, the generalized electric and heat currents, conductivity, Seebeck coefficient, and thermal conductivity are obtained within the isotropic constant relaxation time approximation. To probe experimentally the discussed multiband effects in the thermoelectric properties, one can select materials whose energy band structures have degenerate bands at the Fermi level and apply static external magnetic field. There are numerous such systems discovered and many are considered to be good thermoelctrics with nontrivial topology [26,27]. Thus the material selection can be possible and the application of the static magnetic field can be used to probe the effects arising from the magnetization and Berry curvature properties. Comparing measurements with and without the magnetic field can give an idea to what extent the multiband effects affect the thermoelectric transport.

The developed theory constitutes a significant step forward towards the description of transport by taking into account band structure effects that are not included in the semiclassical single band Boltzmann equation. We emphasize that this approach makes explicit use of multiband wave packets, which is the fundamental difference from the kinetic equation for the Wigner function where wave packets are not used at all. Like the case of $N$ degenerate bands, this approach can be applied to other examples including linear crossing bands, which are of interest to materials with Dirac-like spectra. Additionally, scattering relaxation times and their dependences on energy dispersion and temperature beyond the isotropic model studied here can also be considered. Nevertheless, the purpose of this work is to present this novel theory and emphasize its novelty. Its application to the specific case of $N$ degenerate bands shows that this method can also be applied to other possibly more complicated situations. We point out that this generalized transport theory opens avenues to explore new multiband structure effects in transport.

\section{ACKNOWLEDGMENTS}

L.M.W. acknowledges financial support from the US National Science Foundation under Grant No. DMR-1748188. T.S. acknowledges support from the ERASMUS+ Programme of the European Union and the hospitality of TU Dresden, where some of this research was done. Discussions with Prof. Carsten Timm are also acknowledged.
[1] N. W. Ashcroft and N. D. Mermin, Solid State Physics (Harcourt, 1976), Chaps. 8 and 13.

[2] C. Cercignani, The Boltzmann Equation and its Applications (Springer Science + Business Media, New York, 1988).

[3] D. Xiao, M. C. Chang, and Q. Niu, Rev. Mod. Phys. 82, 1959 (2010).

[4] J. Sinova, S. O. Valenzuela, J. Wunderlich, C. H. Back, and T. Jungwirth, Rev. Mod. Phys. 87, 1213 (2015).
[5] M. C. Chang and Q. Niu, J. Phys. Condens. Matter 20, 193202 (2008).

[6] C. Xiao, Front. Phys. 13, 137202 (2018).

[7] G. Sundaram and Q. Niu, Phys. Rev. B 59, 14915 (1999).

[8] D. Culcer, Y. Yao, and Q. Niu, Phys. Rev. B 72, 085110 (2005).

[9] R. Lundgren, P. Laurell, and G. A. Fiete, Phys. Rev. B 90, 165115 (2014)

[10] K. Y. Bliokh, A. Niv, V. Kleiner, and E. Hasman, Nat. Photon. 2, 748 (2008). 
[11] T. Jungwirth, Q. Niu, and A. H. MacDonald, Phys. Rev. Lett. 88, 207208 (2002).

[12] A. Sekine, D. Culcer, and A. H. MacDonald, Phys. Rev. B 96, 235134 (2017).

[13] R. Shindou and K. I. Imura, Nucl. Phys. B 720, 399 (2005).

[14] C. H. Wong and Y. Tserkovnyak, Phys. Rev. B 84, 115209 (2011).

[15] T. Stedman, C. Timm, and L. M. Woods, New J. Phys. 21, 103007 (2019).

[16] C. L. Fefferman and M. I Watson, Commun. Math. Phys. 326, 251 (2014).

[17] M. Thesberg, H. Kosina, and N. Neophytou, Phys. Rev. B 95, 125206 (2017).

[18] N. P. Armitage, E. J. Mele, and A. Vishwanath, Rev. Mod. Phys. 90, 015001 (2018).

[19] T. O. Wehling, A. M. Black-Schaffer, and A. V. Balatsky, Adv. Phys. 63, 1 (2014).
[20] M. Bonitz, Quantum Kinetic Theory (Springer, Leipzig, 1998).

[21] A. B. Watson, J. F. Lu, and M. I. Weinstein, J. Math. Phys. 58, 021503 (2017).

[22] See Supplemental Material at http://link.aps.org/supplemental/ 10.1103/PhysRevResearch.2.033086 for deriving the density operator at equilibrium, the commutator $[\hat{\rho}, H]$ in the presence of static fields, and the thermoelectric currents for a wave packet composed of $N$ degenerate bands.

[23] M. C. Chang and Q. Niu, Phys. Rev. B 53, 7010 (1996).

[24] J. H. Irving and R. W. Zwanzig, The J. Chem. Phys. 19, 1173 (1951).

[25] J. J. Sakurai and J. Napolitano, Modern Quantum Mechanics (Cambridge University Press, 2017), Chap. 3.4.

[26] L. Muchler, F. Casper, B. Yan, S. Chadov, and C. Felser, Phys. Stat. Solidi RRL 7, 91 (2013).

[27] Y. J. Wang, H. Lin, T. Das, M. Z. Hasan, and A. Bansil, New J. Phys. 13, 085017 (2011). 